\title{
ON THE POISSON REPRESENTATION OF DISTRIBUTIONS
}

\author{
AATOS LAHTINEN
}

\section{Introduction}

1. Let $\mathscr{A}$ be a test function space on $\boldsymbol{R}$ and $\mathscr{A}^{\prime}$ the corresponding space of distributions. The elements of $\mathscr{A}$ and $\mathscr{A}^{\prime}$ are supposed to be complex valued. A complex valued function is called harmonic if its real and imaginary parts are harmonic functions. A distribution $T \in \mathscr{A}^{\prime}$ has a harmonic representation in $\mathscr{B}$ if $\mathscr{B} \subset \mathscr{A}$ and there exists a complex valued function $h$ harmonic in $\boldsymbol{C} \backslash \boldsymbol{R}$ such that

$$
\lim _{\eta \rightarrow 0} \int_{-\infty}^{\infty} h(x+i \eta) \varphi(x) d x=\langle T, \varphi\rangle
$$

for every $\varphi \in \mathscr{B}$. Similarly $T \in \mathscr{A}^{\prime}$ has an analytic representation in $\mathscr{B}$ if $\mathscr{B} \subset \mathscr{A}$ and there exists an analytic function $f$ in $\boldsymbol{C} \backslash \boldsymbol{R}$ such that (1.1) is valid for every $\varphi \in \mathscr{B}$ when $\eta \rightarrow 0+$ and $h(z)=f(z)-f(\bar{z})$.

Let $\mathcal{O}_{\alpha}(\boldsymbol{R})$ be the test function space of functions $\varphi: R \rightarrow C$ such that $\varphi(t)$ together with its derivatives is asymptotically bounded by $|t|^{\alpha}$ as $|t| \rightarrow \infty$. In [2] Bremermann has shown, among other results, that $T \in \mathscr{E}^{\prime}(\boldsymbol{R})$ has a harmonic representation in $\mathcal{O}_{0}(\boldsymbol{R})$ and that $T \in \mathcal{O}_{\alpha}^{\prime}(\boldsymbol{R}), \alpha \geqq-1$, has an analytic representation in $\mathscr{D}(\boldsymbol{R})$.

In this paper we consider the harmonic representation of $T \in \mathcal{O}_{\alpha}^{\prime}(\boldsymbol{R})$ in $\mathcal{O}_{\alpha}(\boldsymbol{R})$ following Bremermann. We mainly use the notations and terminology of [2]. For the basic properties of distributions we also refer to Schwartz [5]. Let us denote the Poisson kernel by $p(t, z)$, i.e.,

$$
p(t, z)=\frac{1}{\pi}|y| \cdot|t-z|^{-2}, \quad z=x+i y .
$$

The function $p(\cdot, z)$ belongs to $\mathcal{O}_{\alpha}(\boldsymbol{R})$ for every $z \in \boldsymbol{C} \backslash \boldsymbol{R}$ if $\alpha \geqq-2$. For those values of $\alpha$ we can define for every $T \in \mathcal{O}_{\alpha}^{\prime}(\boldsymbol{R})$ a complex valued function $z \rightarrow\langle T, p(\cdot, z)\rangle$ which is harmonic in $\boldsymbol{C} \backslash \boldsymbol{R}$. We call this function the Poisson representation of $T$, and show that it gives a harmonic representation of $T \in \mathcal{O}_{\alpha}^{\prime}(\boldsymbol{R})$ in $\mathcal{O}_{\alpha}(\boldsymbol{R})$ if $-2 \leqq \alpha<1$. As an immediate corollary we get an analytic representation of $T \in \mathcal{O}_{\alpha}^{\prime}(\boldsymbol{R})$ in $\mathcal{O}_{\alpha}(\boldsymbol{R})$ if $-1 \leqq \alpha<1$. 


\section{Asymptotically bounded functions}

2. Let $\alpha \in \boldsymbol{R}$. A complex valued function $f: t \rightarrow f(t), t \in \boldsymbol{R}$, is called asymptotically bounded by $|t|^{\alpha}$ and denoted by $f(t)=\mathcal{O}\left(|t|^{\alpha}\right)$ if there exist non-negative constants $c$ and $r$, asymptotic constants, such that $|f(t)| \leqq c|t|^{\alpha}$ if $|t| \geqq r$. We call $\alpha$ the asymptotic degree of $f$. The set of non-negative integers is denoted by $\boldsymbol{Z}_{+}$.

If $a, b \in \boldsymbol{R} \cup\{ \pm \infty\}, z \in \boldsymbol{C}$, and the function $f$ is defined on $(a, b)$, we denote

$$
P I(f, z ; a, b)=\int_{a}^{b} p(t, z) f(t) d t,
$$

provided that the integral exists. Here $p(t, z)$ is the Poisson kernel defined in (1.2). We also denote

$$
P I(f, z)=P I(f, z ;-\infty, \infty) .
$$

Using the Poisson integral we can represent asymptotically bounded functions as boundary values of harmonic functions.

Proposition 2.1. Let $f \in C^{m}(\boldsymbol{R})$ and $D^{k} f(t)=\mathcal{O}\left(|t|^{\alpha}\right)$ for every $k \in \boldsymbol{Z}_{+}, 0 \leqq k \leqq m$. If $\alpha<1$, then

$$
\lim _{\eta \rightarrow 0} D^{k} P I(f, x+i \eta)=D^{k} f(x), \quad 0 \leqq k \leqq m,
$$

uniformly in compact subsets of $\boldsymbol{R}$.

Propositions of this kind are usually proved on the assumption that $\alpha=0$ (cf. [2] and [4]). The proof of this slightly more general form is essentially the same. One only has to be more careful with estimates because of the growth of $f$ at infinity.

We say that $P(f, z)$ is the Poisson representation of $f$. It should be noted that

$$
P I(1, z)=1
$$

and that

$$
D_{x}^{k} P I(f, z)=P I\left(D^{k} f, z\right) .
$$

3. Let $\beta<1$ and let $f \in C(\boldsymbol{R}), f(t)=\mathcal{O}\left(|t|^{\beta}\right)$. Then $P I(f, x+i \eta)$, the Poisson representation of $f$, exists and is a continuous function of $x$ for fixed values of $\eta \in \boldsymbol{R} \backslash\{0\}$. We intend to study the asymptotical boundedness of $P I(f, x+i \eta)$ as a function of $x$. To be more exact, we want to know the asymptotic degree and, moreover, in which cases the asymptotic constants can be chosen to be independent of $\eta$. This information is needed in the next chapter.

Proposition 2.2. Let $f \in C(R), f(t)=\mathcal{O}\left(|t|^{\beta}\right), \beta<1$, and let $\alpha \geqq \max (\beta,-2)$. If $\eta_{0}>0$ is given and $0<|\eta| \leqq \eta_{0}$, then $P I(f, x+i \eta)=\mathcal{O}\left(|x|^{\alpha}\right)$, and the asymptotic constants can be chosen to be independent of $\eta$. 
Proof. Let $\eta_{0}>0$ and $0<|\eta| \leqq \eta_{0}$. Let $c$ and $r$ be asymptotic constants of $f$. We divide the Poisson representation of $f$ into three parts, $P I(f, \cdot)=P I(f, \cdot ;-r, r)+$ $P I(f, \cdot ; r, \infty)+P I(f, \cdot ;-\infty,-r)$, and estimate each part separately.

For the first term we get directly an upper bound

$$
|P I(f, x+i \eta ;-r, r)| \leqq \frac{8 r \eta_{0}}{\pi} \sup _{-r \leqq t \leqq r}|f(t)| \cdot|x|^{-2} \quad \text { if } \quad|x| \geqq 2 r .
$$

This has the required asymptotic behaviour.

The remaining two terms appear to have similar upper bounds

$$
\begin{aligned}
|P I(f, x+i \eta ; r, \infty)| & \leqq c P I\left(t^{\beta}, x+i \eta ; r, \infty\right) \quad \text { if } \quad|x| \geqq r, \\
|P I(f, x+i \eta ;-\infty,-r)| & \leqq c P I\left(t^{\beta},-x+i \eta ; r, \infty\right) \quad \text { if } \quad|x| \geqq r .
\end{aligned}
$$

Thus the proposition is proved if we show that there exist non-negative constants $c_{0}$ and $r_{0}$ such that

$$
P I\left(t^{\beta}, x+i \eta ; r, \infty\right) \leqq c_{0}|x|^{\alpha}, \quad \text { if } \quad|x| \geqq r_{0},
$$

for every $\eta \in \boldsymbol{R}, 0<|\eta| \leqq \eta_{0}$.

If $\beta$ is not an integer, (2.7) can be obtained by means of residue calculus. So we suppose first that $\beta<1, \beta \notin Z$, and consider a value of $x$ for which $|x| \geqq 2 r+4 \eta_{0}$. We imbed the $t$-axis into the complex plane $C_{w}, w=t+i u$, choose a constant $R$ such that $R \geqq 2|x|+4 \eta_{0}$, and define paths $\gamma_{j}:[0,1] \rightarrow C_{w}$ as follows: $\gamma_{1}: s \rightarrow R e^{i 2 \pi s}$, $\gamma_{2}: s \rightarrow(R+s(r-R)) e^{i 2 \pi}, \gamma_{3}: s \rightarrow r e^{i 2 \pi(1-s)}, \gamma_{4}: s \rightarrow r+s(R-r)$. We also define $\gamma_{0}=\gamma_{1}+$ $\gamma_{2}+\gamma_{3}+\gamma_{4}$. Along these paths we define integrals

$$
I_{j}=\frac{|\eta|}{\pi} \int_{\gamma_{j}} \frac{w^{\beta}}{(w-x)^{2}+\eta^{2}} d w, \quad j=0,1,2,3,4 .
$$

Here the argument of $w^{\beta}$ is taken between 0 and $2 \pi \beta$. Because $I_{2}$ and $I_{4}$ are of the type of (2.1) we get a representation

$$
P I\left(t^{\beta}, x+i \eta ; r, R\right)=\left(1-e^{2 \pi i \beta}\right)^{-1}\left(I_{0}-I_{1}-I_{3}\right) .
$$

In order to get (2.7) we estimate each integral of the right side separately.

The value of $I_{0}$ is equal to the sum of the residues of the integrand multiplied by $2 \pi i$ (cf. e.g. [1]). Because the integrand has only two simple poles at $x \pm i \eta$, $I_{0}=\operatorname{sgn}(\eta)\left[(x+i \eta)^{\beta}-(x-i \eta)^{\beta}\right]$. This gives easily an estimate of the right kind

$$
\left|I_{0}\right| \leqq 4|x|^{\beta} \quad \text { if } \quad|x| \geqq 2 r+4 \eta_{0} .
$$

A straightforward calculation gives an estimate of $I_{1}$ in $R$,

$$
\left|I_{1}\right| \leqq 16 \eta_{0} R^{\beta-1} \quad \text { if } \quad R \geqq 2|x|+4 \eta_{0} .
$$

Because $\beta<1, I_{1}$ tends to zero as $R$ tends to infinity. In the same way we can estimate $I_{3}$ in $x$,

$$
\left|I_{3}\right| \leqq 16 \eta_{0} r^{\beta+1}|x|^{-2} \quad \text { if } \quad|x| \geqq 2 r+4 \eta_{0} .
$$


If we take absolute values of both sides of (2.9), use the estimates (2.10), (2.11), (2.12), and let $R$ tend to infinity, we get an estimate of the type of (2.7) with values $c_{0}=\left|1-e^{2 \pi i \beta}\right|^{-1}\left(4+16 \eta_{0} r^{\beta+1}\right)$ and $r_{0}=2 r+4 \eta_{0}$. These values are clearly independent of $\eta$. So (2.7) is proved in the case $\beta \notin Z$.

For integer values of $\beta$ (2.7) can be obtained by direct integration and estimation. If $\beta=0$, then $\alpha \geqq 0$ and the statement follows from (2:3). In the cases $\beta=-1$ and $\beta=-2$ we get the same final estimate

$$
P I\left(t^{\beta}, x+i \eta ; r, \infty\right) \leqq\left(\eta_{0}+1\right)|x|^{\beta} \quad \text { if } \quad|x| \geqq 2 r \geqq 2 .
$$

This shows (2.7) for values $\beta=-1,-2$. If finally $\beta \in \boldsymbol{Z}, \beta<-2$, then $\alpha \geqq-2$ and $P I\left(t^{\beta}, x+i \eta ; r, \infty\right) \leqq P I\left(t^{-2}, x+i \eta ; r, \infty\right)$ when $r \geqq 1$. This implies (2.7) for the remaining values of $\beta$ by (2.13). The proposition is proved.

\section{The Poisson representation of distributions}

4. We define the space of asymptotically bounded test functions as in [2]: If $\alpha \in \boldsymbol{R}$, then

$$
\mathcal{O}_{\alpha}(\boldsymbol{R})=\left\{\varphi \in C^{\infty}(\boldsymbol{R}) \mid D^{k} \varphi(t)=\mathcal{O}\left(|t|^{\alpha}\right) \text { for every } k \in \boldsymbol{Z}_{+}\right\} .
$$

If $\alpha \leqq \beta$, then $\mathscr{D}(\boldsymbol{R}) \subset \mathcal{O}_{\alpha}(\boldsymbol{R}) \subset \mathcal{O}_{\beta}(\boldsymbol{R}) \subset \mathscr{E}(\boldsymbol{R})$. Using Propositions 2.1 and 2.2 together with (2.4) we get the following result:

Proposition 3.1. Let $\varphi \in \mathcal{O}_{\beta}(\boldsymbol{R}), \beta<1$ and let $\alpha \geqq \max (\beta,-2)$. If $\eta \in \boldsymbol{R} \backslash\{0\}$, then $P I(\varphi, x+i \eta) \in \mathcal{O}_{\alpha}(\boldsymbol{R})$ as a function of $x$ and

$$
\lim _{\eta \rightarrow 0} P I(\varphi, x+i \eta)=\varphi(x)
$$

in $\mathcal{O}_{\alpha}(\boldsymbol{R})$.

5. The distribution space corresponding to $\mathcal{O}_{\alpha}(\boldsymbol{R})$ is denoted by $\mathcal{O}_{\alpha}^{\prime}(\boldsymbol{R})$. It consists of continuous linear functionals $T: \mathcal{O}_{\alpha}(\boldsymbol{R}) \rightarrow \boldsymbol{C}$. The value of a distribution $T$ applied to a test function $\varphi$ is denoted by $\langle T, \varphi\rangle$. It is easily seen that the Poisson kernel $p(\cdot, z)$ belongs to $\mathcal{O}_{\alpha}(\boldsymbol{R})$ for every $z \in \boldsymbol{C} \backslash \boldsymbol{R}$ if $\alpha \geqq-2$. Thus we can define a function

$$
P I(T, z)=\langle T, p(\cdot, z)\rangle
$$

for every $T \in \mathcal{O}_{\alpha}^{\prime}(\boldsymbol{R}), \alpha \geqq-2$. We call this function the Poisson representation of $T$. The harmonicity of the Poisson kernel implies that also the Poisson representation is a harmonic function outside the real axis.

Proposition 3.2. If $T \in \mathcal{O}_{\alpha}^{\prime}(R), \alpha \geqq-2$, then its Poisson representation $\operatorname{PI}(T, \cdot)$ is harmonic in $\boldsymbol{C} \backslash \boldsymbol{R}$. 
Proof. We begin by showing that

$$
D_{x} P I(T, z)=\left\langle T, D_{x} p(\cdot, z)\right\rangle .
$$

Suppose that $z \in \boldsymbol{C} \backslash \boldsymbol{R}, t \in \boldsymbol{R}$ and $h \in \boldsymbol{R} \backslash\{0\}$. Consider the difference

$$
A_{x}(t, h)=\frac{1}{h}(p(t, z+h)-p(t, z))-D_{x} p(t, z) .
$$

If $n \in \boldsymbol{Z}_{+}$, we can show by induction that

$$
D_{t}^{n} A_{x}(t, h)=h B_{n}(t, z, h)\left(|t-z-h|^{2}|t-z|^{4}\right)^{-n-1},
$$

where $B_{n}$ is a polynomial of $t, z$ and $h$. As a polynomial of $t$ the degree of $B_{n}$ is at most $5 n+2$. Thus (3.6) implies that $D_{t}^{n} A_{x}(t, h)$ converges to zero uniformly on compact sets as $h \rightarrow 0$. Moreover, if $h_{0}>0$ is small enough, then $D_{t}^{n} A_{x}(t, h)=$ $\mathcal{O}\left(|t|^{-2}\right)$ and the asymptotic constants are independent of $h$ when $0<|h| \leqq h_{0}$. Therefore

$$
\lim _{h \rightarrow 0} A_{x}(t, h)=0
$$

in $\mathcal{O}_{\alpha}(\boldsymbol{R}), \alpha \geqq-2$. This proves (3.4).

The rest of the proof runs along the same line. If we replace $p$ by $D_{x} p$ in (3.4), we see in a similar way that (3.7) is still valid in $\mathcal{O}_{\alpha}(\boldsymbol{R}), \alpha \geqq-2$. Thus

$$
D_{x}^{2} P I(T, z)=\left\langle T, D_{x}^{2} p(\cdot, z)\right\rangle .
$$

Proceeding to the $y$-direction in the same way we see that (3.8) remains valid if $D_{x}^{2}$ is replaced by $D_{y}^{2}$. Because $p(t, z)$ is harmonic in $\boldsymbol{C} \backslash \boldsymbol{R}$ these facts imply that $\left(D_{x}^{2}+D_{y}^{2}\right) \operatorname{PI}(T, z)=\left\langle T,\left(D_{x}^{2}+D_{y}^{2}\right) p(\cdot, z)\right\rangle=0$ if $z \in \boldsymbol{C} \backslash \boldsymbol{R}$. The proposition is proved.

6. The Poisson representation of a distribution $T$ can be used for the characterization of $T$ as follows:

Proposition 3.3. Let $\beta<1$ and $\alpha \geqq \max (\beta,-2)$. If $T \in \mathcal{O}_{\alpha}^{\prime}(\boldsymbol{R})$ and $\varphi \in \mathcal{O}_{\beta}(\boldsymbol{R})$, then

$$
\lim _{\eta \rightarrow 0} \int_{-\infty}^{\infty} P I(T, x+i \eta) \varphi(x) d x=\langle T, \varphi\rangle .
$$

Proof. Because $\beta \leqq \alpha$, we have $\varphi \in \mathcal{O}_{\alpha}(\boldsymbol{R})$ and the right side of (3.9) is well defined. We consider the integral on the left side. Let $a<0<b$ and let $\left\{x_{j}\right\}_{-n}^{n}$ be a division of the interval $(a, b)$ into equal subintervals. We show that if $\eta \in \boldsymbol{R} \backslash\{0\}$, then

$$
\lim _{n \rightarrow \infty} \sum_{j=-n}^{n} \varphi\left(x_{j}\right) p\left(t, x_{j}+i \eta\right) \Delta x_{j}=P I(\varphi, t+i \eta ; a, b)
$$

in $\mathcal{O}_{\alpha}(\boldsymbol{R})$. Denote the sum of the left side by $\sigma_{n}$. If $m \in \boldsymbol{Z}_{+}$, it is not difficult to see that

$$
\lim _{n \rightarrow \infty} D_{t}^{m} \sigma_{n}=D_{t}^{m} P I(\varphi, t+i \eta ; a, b)
$$


uniformly in compact sets. If $c$ and $r, r \geqq 2 \max (-a, b)$, are asymptotic constants of $D_{t}^{m} p\left(t, x_{j}+i \eta\right)$, we can suppose that they are independent of the division of the interval $(a, b)$. Thus the estimate

$$
\left|D_{t}^{m} \sigma_{n}\right| \leqq(b-a) \cdot \sup _{x \in(a, b)}|\varphi(x)| \cdot c \cdot|t|^{\alpha}, \quad \text { if } \quad|t| \geqq r,
$$

gives the right asymptotic boundedness independent of $n$. So (3.10) is valid in $\mathscr{O}_{\alpha}(\boldsymbol{R})$. This means that

$$
\begin{gathered}
\int_{a}^{b} P I(T, x+i \eta) \varphi(x) d x=\lim _{n \rightarrow \infty} \sum_{j=-n}^{n} P I\left(T, x_{j}+i \eta\right) \varphi\left(x_{j}\right) \Delta x_{j} \\
=\lim _{n \rightarrow \infty} \sum_{j=-n}^{n}\left\langle T_{t}, p\left(t, x_{j}+i \eta\right) \varphi\left(x_{j}\right) \Delta x_{j}\right\rangle=\left\langle T_{t}, P I(\varphi, t+i \eta ; a, b)\right\rangle .
\end{gathered}
$$

Next we show that

$$
\lim _{-a, b \rightarrow \infty} P I(\varphi, t+i \eta ; a, b)=P I(\varphi, t+i \eta)
$$

in $\mathcal{O}_{\alpha}(\boldsymbol{R})$. If $m \in \boldsymbol{Z}_{+}$, it is not difficult to see that

$$
\lim _{-a, b \rightarrow \infty} D_{t}^{m} P I(\varphi, t+i \eta ; a, b)=D_{t}^{m} P I(\varphi, t+i \eta)
$$

uniformly in compact sets. It remains to show the asymptotic boundedness. The derivative $D_{t}^{m} p(x, t+i \eta)$ can be written in the form $B_{m}(t, x) \cdot p(x, t+i \eta)$, where the function $B_{m}: \boldsymbol{R} \times \boldsymbol{R} \rightarrow \boldsymbol{R}$ is bounded. If $d$ is an upper bound of $\left|B_{m}\right|$, if $c$ and $r$ are asymptotic constants of $\varphi$ and if $-a \geqq r, b \geqq r$, then we get an estimate

$$
\begin{gathered}
\left|D_{t}^{m}(P I(\varphi, t+i \eta)-P I(\varphi, t+i \eta ; a, b))\right| \\
\leqq c d\left(P I\left(|x|^{\beta}, t+i \eta ; r, \infty\right)+P I\left(|x|^{\beta},-t+i \eta ; r, \infty\right)\right) .
\end{gathered}
$$

By Proposition 2.2 there exist constants $c_{0}$ and $r_{0}$ such that

$$
P I\left(|x|^{\beta}, t+i \eta ; r, \infty\right) \leqq c_{0}|t|^{\alpha} \quad \text { if } \quad|t| \geqq r_{0}
$$

for every $r \geqq r_{0}$. This fact together with the preceding estimate gives the right asymptotic boundedness. So (3.12) is valid in $\mathscr{O}_{\alpha}(\boldsymbol{R})$. Together with (3.11) it shows that

$$
\int_{-\infty}^{\infty} P I(T, x+i \eta) \varphi(x) d x=\left\langle T_{t}, P I(\varphi, t+i \eta)\right\rangle .
$$

The statement (3.9) follows now from (3.13) by Proposition 3.1. The proposition is proved.

7. Now we can formulate our main result. We recall that a distribution $T \in \mathcal{O}_{\alpha}^{\prime}(\boldsymbol{R})$ has a harmonic representation in $\mathcal{O}_{\alpha}(\boldsymbol{R})$ if there exists a harmonic function $h: \boldsymbol{C} \backslash \boldsymbol{R} \rightarrow \boldsymbol{C}$ such that

$$
\lim _{\eta \rightarrow 0} \int_{-\infty}^{\infty} h(x+i \eta) \varphi(x) d x=\langle T, \varphi\rangle
$$


for every $\varphi \in \mathcal{O}_{\alpha}(\boldsymbol{R})$. The Poisson representation provides us a simple means of obtaining a harmonic representation in some cases. In fact, Propositions 3.2 and 3.3 give us immediately:

Theorem 1. Let $T \in \mathcal{O}_{\alpha}^{\prime}(R),-2 \leqq \alpha<1$. Then $P I(T, \cdot)$ is a harmonic representation of $T$ in $\mathcal{O}_{\alpha}(\boldsymbol{R})$.

\section{The Cauchy representation of distributions}

8. Let $\alpha \geqq-1$ and $T \in \mathcal{O}_{\alpha}^{\prime}(\boldsymbol{R})$. The function

$$
\hat{T}(z)=\frac{1}{2 \pi i}\left\langle T_{t}, \frac{1}{t-z}\right\rangle
$$

is called the Cauchy representation of $T$. It is an analytic function of $z$ in $C \backslash s p t T$ (cf. [2]). Because $\hat{T}(z)-\hat{T}(\bar{z})=\operatorname{sgn}(\operatorname{Im} z) P I(T, z)$ we have by Proposition 3.3

$$
\lim _{\eta \rightarrow 0_{+}} \int_{-\infty}^{\infty}(\hat{T}(x+i \eta)-\hat{T}(x-i \eta)) \varphi(x) d x=\langle T, \varphi\rangle
$$

for every $\varphi \in \mathcal{O}_{\alpha}(\boldsymbol{R})$ if $-1 \leqq \alpha<1$. In other words, the following result is valid:

Theorem 2. Let $T \in \mathcal{O}_{\alpha}^{\prime}(\boldsymbol{R}),-1 \leqq \alpha<1$. Then $\hat{T}$ is an analytic representation of $T$ in $\mathcal{O}_{\alpha}(\boldsymbol{R})$.

\section{References}

[1] Ahlfors, L. V.: Complex analysis. - 2nd ed., McGraw-Hill Book Company, New YorkSt. Louis-San Francisco-Toronto--London-Sydney, 1966.

[2] Bremermann, H. J.: Distributions, complex variables and Fourier transforms. - Addison-Wesley Publishing Company, Inc., Reading, Massachusetts-Palo Alto-London-New York-Dallas-Atlanta-Barrington, Illinois, 1965.

[3] Bremermann, H. J., and L. Durand: On analytic continuation, multiplication, and Fourier transformations of Schwartz distributions. - J. Mathematica! Phys. 2, 1961, 240 258.

[4] Helms, L. L.: Introduction to potential theory. - Wiley-Interscience, New York-LondonSydney-Toronto, 1969.

[5] Schwartz, L.: Théorie des distributions. - 3rd ed,. Hermann et $C^{\text {ie }}$, Paris, 1966.

University of Helsinki

Department of Mathematics

SF-00100 Helsinki 10

Finland

Received 7 November 1978

Revision received 26 March 1979 